\title{
Análise Postural dos Praticantes de Kickboxing
}

\author{
Postural Analysis of Kickboxing Practicers
}

Análisis Postural de los Practicantes de Kickboxing

\author{
Alysson Jemesson dos Santos \\ Walmir Romário dos Santos \\ Pedro Pinheiro Paes \\ Wlaldemir Roberto dos Santos ${ }^{1,4}$
}

\section{Resumo}

O Kickboxing é uma modalidade esportiva individual de combate que exige em sua técnica, a execução de movimentos em grandes amplitudes, equilíbrio, agilidade, resistência muscular e aeróbia, para isso se faz necessário uma dedicação de frequência de programas de treinamentos e intensidade na iniciação esportiva. Tal frequência e intensidade podem predispor aos atletas possíveis índices de lesões musculoesqueléticas e alterações posturais no decorrer de sua prática. O presente estudo teve como objetivo detectar assimetrias e possíveis desvios posturais, além de interpretar as regiões mais acometidas nos praticantes da modalidade do Kickboxing através de um questionário e do protocolo proposto pela Portland State University (PSU), por meio de fotogrametria, onde o valor de índice de correção postural (ICP) considerado é $\geq 65 \%$ para indivíduos adultos. A amostra foi composta por 17 praticantes de Kickboxing do sexo masculino, com média de idade de 24,3 anos, com no mínimo 6 meses de prática. Os resultados obtiveram um ICP no valor de 85,9\%, como parâmetro acima de normalidade sugerido pelo método PSU. Entretanto, percebeu-se em uma análise por segmento que todos os indivíduos $(100 \%)$ apresentavam alteração na altura dos ombros, no tronco $(70,8 \%)$, posição da cabeça $(58,8 \%)$ e alterações no pé $(41,2 \%)$. Desta forma, observou-se que o padrão exigido pela prática do Kickboxing pode levar a uma postura alterada, aumentando o risco de lesões e reduzindo o desempenho. Com isso, verificamos a importância com o cuidado da postura dos praticantes de Kickboxing; na perspectiva de melhorar o desempenho e reduzir o risco de lesões.

Palavras - chave: Análise Postural. Kickboxing. Exercício Físico.

\footnotetext{
Abstract

Kickboxing is an individual sport fighting mode that require in its technique, the execution of movements in great amplitudes, balance, agility, muscular resistance and aerobic, for that it is necessary a dedication of frequency of training programs and intensity in the sports initiation. Such frequency and intensity may predispose athletes to possible rates of musculoskeletal injuries and postural changes in the course of their practice. The present study aimed to detect asymmetries and possible postural deviations, as well as to interpret the most affected regions in Kickboxing practitioners through a questionnaire and the protocol proposed by the Portland State University (PSU), by means of photogrammetry, where the value of postural correction index (PCI) considered is $\geq 65 \%$ for adult individuals. The sample consisted of 17 male Kickboxing practitioners, with a mean age of 24.3 years, with at least 6 months of practice. The results obtained an ICP of $85.9 \%$, as a parameter above normality suggested by the PSU

1. Centro Universitário FBV Wyden - UNIFBV/Wyden, Recife, Pernambuco, Brasil. 2. Universidade de São Paulo - EEFERP/USP, Ribeirão Preto, São Paulo, Brasil. 3. Universidade Federal de Pernambuco - DEF/UFPE, Recife, Pernambuco, Brasil. 4. Centro Universitário FAVIP Wyden - UNIFAVIP/Wyden, Caruaru, Pernambuco, Brasil. Autor para correspondência: Wlaldemir Roberto dos Santos, Centro Universitário FBV Wyden - UNIFBV Wyden, Recife, Pernambuco, Rua Jean-Émile Favre, 422, 51190-450, Imbiribeira, Recife, PE, Brasil, e-mail: wlaldemir@hotmail.com . Este conteúdo utiliza a Licença Creative Commons Attribution 4.0 International License Open Access. This content is licensed under a Creative Commons attribution-type BY. ISSN 2595 - 0096.
} 
method. However, it was observed in a segmental analysis that all individuals $(100 \%)$ had altered shoulder height, trunk (70.8\%), head position (58.8\%) and foot changes (41.2\%). In this way, it was observed that the standard required by the practice of Kickboxing can lead to an altered posture, increasing the risk of injuries and reducing performance. With this, we note the importance of taking care of the posture of Kickboxing practitioners, with a view to improving performance and reducing the risk of injury.

Keywords: Postural Analysis. Kickboxing. Physical Exercise.

\section{Resumen}

El Kickboxing es una modalidad deportiva individual de combate que exige en su técnica, la ejecución de movimientos en grandes amplitudes, equilibrio, agilidad, resistencia muscular y aeróbica, para ello se hace necesaria una dedicación de frecuencia de programas de entrenamientos e intensidad en la iniciación deportiva. Tal frecuencia e intensidad pueden predisponer a los atletas posibles índices de lesiones musculoesqueléticas y alteraciones posturales en el transcurso de su práctica. El presente estudio tuvo como objetivo detectar asimetrías y posibles desvíos posturales, además de interpretar las regiones más acometidas en los practicantes de la modalidad del Kickboxing a través de un cuestionario y del protocolo propuesto por la Portland State University (PSU), por medio de fotogrametría, donde el valor de índice de corrección postural (ICP) considerado es $\geq 65 \%$ para los adultos. La muestra fue compuesta por 17 practicantes de Kickboxing del sexo masculino, con una media de edad de 24,3 años, con al menos 6 meses de práctica. Los resultados obtuvieron un ICP en el valor del 85,9\%, como parámetro por encima de la normalidad sugerido por el método PSU. Sin embargo, se percibió en un análisis por segmento que todos los individuos $(100 \%)$ presentaban alteración en la altura de los hombros, en el tronco $(70,8 \%)$, posición de la cabeza $(58,8 \%)$ y alteraciones en el pie (41,2\%). De esta forma, se observó que el estándar exigido por la práctica del Kickboxing puede llevar a una postura alterada, aumentando el riesgo de lesiones y reduciendo el desempeño. Con ello, verificamos la importancia con el cuidado de la postura de los practicantes de Kickboxing, en la perspectiva de mejorar el desempeño y reducir el riesgo de lesiones.

Palabras - clave: Análisis Postural. Kickboxing. Ejercicio físico.

\section{INTRODUÇÃO}

O Kickboxing é uma modalidade de luta executada em pé, necessitando de uma boa condição postural do corpo para a sua prática. Consiste em chutes e socos, com um diferencial de ter como aspecto principal também as técnicas de projeções e quedas, presentes nas artes marciais chinesas ou japonesas'. Deste modo, a exigência da condição física para praticantes de kickboxing é alta, com programas de treinamento que necessitam de ganhos de velocidade de movimento, força, flexibilidade, resistência muscular e aeróbia, capacidades que são de grande importância para suportar o desgaste físico e a aplicação de técnicas, além de serem pontos importantes para prevenir lesões e desvios posturais, tanto no treinamento quanto no momento competitivo ${ }^{2}$.

Poucos são os estudos realizados associando a postura com a prática desportiva, ainda mais quando se trata de Kickboxing. Sabe-se que a postura é 
considerada uma interação das posições das diferentes articulações do corpo em um determinado momento ${ }^{3}$, sendo assim, valências fisiológicas e biomecânicas associadas a um alinhamento postural adequado, tem como objetivo minimizar os estresses e as sobrecargas sofridas a ossos e músculos, protegendo estruturas corporais que entram em desgastes provocados pelos efeitos deletérios do envelhecimento, bases patológicas, treinamento e periodização inadequada ${ }^{4}$.

Durante o treinamento de kickboxing os indivíduos tendem a utilizar a musculatura anterior mais do que a musculatura posterior do corpo, considerando que a maioria das técnicas está associada a esse gesto motor, o que pode causar encurtamentos, prejuízos à postura e desequilíbrio muscular ${ }^{5}$.

Desta maneira, os gestos específicos do esporte e os erros na técnica de execução dos movimentos podem aumentar a prevalência dos desvios posturais e, consequentemente, aumentar o índice de lesões ${ }^{6}$ Entretanto, as modificações desencadeadas pelo treinamento orientado por um profissional incluem ganhos significativos e adaptações físicas, como melhora da capacidade funcional e da postura na prática desportiva $^{7}$. Deste modo, o presente estudo teve como intuito identificar alterações posturais em praticantes de kickboxing.

\section{MÉTODOS}

Trata-se de um estudo observacional, que foi realizado com praticantes de Kickboxing do sexo masculino, de uma equipe da zona sul do município de Recife/PE/Brasil, em Janeiro de 2018. Onde os procedimentos deste estudo seguiram todas as normas éticas estipuladas de acordo com a resolução 466/12 que regulamenta a pesquisa envolvendo seres humanos, sendo $\mathrm{O}$ trabalho aprovado pelo comitê de ética e pesquisa (CAE: 58888516.4.0000.5380).

Foram incluídos no estudo homens que treinavam Kickboxing há pelo menos seis meses, que apresentaram boa condição de saúde (com atestado médico liberatório para a prática) e que tivessem entre 18 a 35 anos. A seleção foi realizada por convite pessoal, de 50 praticantes de Kickboxing de uma equipe da zona sul do município de Recife/Pernambuco/Brasil, os que se enquadraram nos critérios de inclusão supracitados e aceitaram participar, assinando o termo de consentimentos livre e esclarecido.

De maneira anônima, os participantes responderam a um questionário sobre o tempo da prática do kickboxing, outras práticas de exercícios físicos e motivo por ter escolhido essa 
modalidade de luta. Para realizar a avaliação postural, utilizamos o protocolo proposto pela Portland State University $(\mathrm{PSU})^{8}$. O protocolo permite, por meio de observação subjetiva, quantificar e avaliar o Índice de Correção Postural (ICP), utilizando-se de equações matemáticas que são estipuladas pelo score diagnóstico, sendo analisado de modo geral e subdividido por regiões corporais, detectando as assimetrias e os possíveis desvios posturais entre os segmentos corporais dos avaliados. Para amenizar os erros intra-avaliadores durante os testes, todas as análises foram realizadas pelo primeiro autor do presente estudo.

O PSU foi utilizado para determinar o ICP, que consiste em uma escala determinada por figuras-modelos de 15 segmentos corporais, RCP - região da cabeça e do pescoço (5 regiões), RCDL região da coluna dorsal e lombar (3 regiões), RAQ - região do abdômen e quadril (3 regiões), RMI - região de membros inferiores (4 regiões). Cada segmento apresenta um score para o desvio verificado, o item “desvio acentuado” valerá um ponto, o item "ligeiro desvio", três pontos e o item "sem desvio", cinco pontos, que serão utilizados para definir o score final da avaliação. Após a mensuração, foi definido o índice de correção postural (ICP), no qual indivíduos adultos com ICP acima de $65 \%$ possuem uma boa postura corporal $^{8}$.

$$
\text { Para realização da avaliação }
$$
postural, os indivíduos estavam vestindo calção de banho ou short segunda pele, sendo colocados em posição ortostática, com pés ligeiramente abduzidos e membros superiores estendidos ao lado do corpo. Para servir como referência dos pontos mesurados foi utilizado um fio de prumo, onde coincidia com uma posição ligeiramente anterior ao maléolo lateral e ao eixo da articulação do joelho, ligeiramente posterior ao eixo da articulação do quadril, corpos das vértebras lombares, articulação do ombro, corpos da maioria das vértebras cervicais, meato auditivo externo e ligeiramente posterior ao ápice da sutura coronal, sendo fixado em um suporte de um metro de distância da parede. Na vista posterior a linha de prumo será equidistante das faces mediais dos calcanhares, pernas e coxas, escápulas e coincidirá com a linha mediana do tronco e cabeça ${ }^{9}$. Toda a avaliação foi registrada por uma câmera fotográfica digital da marca Canon EOS Rebel XS 1000d, lente 18$55 \mathrm{~mm}$, com um tripé posicionado paralelo ao chão com a câmera a uma altura de um metro do chão. Os indivíduos ficaram em um local previamente demarcado, com uma distância padronizada de três metros da câmera fotográfica em uma sala bem 
iluminada com a parede branca não reflexiva.

Foram realizados dois registros fotográficos para cada indivíduo, que foram posicionados em pé, na vista dorsal e lateral, tendo como parâmetro a demarcação do score diagnóstico em cada região de acordo com o método. Após a avaliação, os dados foram anotados em um instrumento de coleta de dados, computados em uma planilha no Microsoft Excel versão 2013 e submetidos a uma análise estatística descritiva para determinar a caracterização da mostra e o ICP, para verificar a heterogenia da amostra foi realizado $\mathrm{um}$ teste $\mathrm{t}$ de amostras independentes (student) com nível de significância estabelecida em p >0,05.

\section{RESULTADOS E DISCUSSÃO}

Avaliou-se a postura corporal estática de 17 praticantes de kickboxing do sexo masculino, com média de idade de $24,3 \pm 5,2$ anos, estatura de $174,5 \pm 0,1 \mathrm{~cm} \mathrm{e}$ massa corporal de 80,41 $\pm 20,25 \mathrm{~kg}$. Os avaliados possuíam em média 22,6 \pm 29,0 meses de prática do kickboxing, com frequência média de 4,6 \pm 1,5 por semana, treinando 82,9 $\pm 32,7 \mathrm{~min} /$ dia (Tabela 1 ). Verificando a heterogenia do grupo apresentou diferença significante em idade, tempo de prática e minutos de prática por dia. Referente a lesões, apenas dois $(11,8 \%)$ praticantes relataram ter lesões ostemioarticulares, lesão no menisco e hérnia torácica. Quanto a prática esportiva, nove participantes $(70,6 \%)$ relataram realizar outro tipo de atividade além do kickboxing.

Tabela 1. Caracterização da amostra $(n=17)$.

\begin{tabular}{lr} 
Variáveis & $\begin{array}{r}\text { Média } \pm \text { Desvio Padrão } \\
\text { (Mínimo - Máximo) }\end{array}$ \\
\hline Idade (anos) & $24,3 \pm 5,2(18,0-35,0){ }^{*}$ \\
Peso (Quilogramas) & $80,4 \pm 20,2(51,0-138,0)$ \\
Estatura (Centímetros) & $174,5 \pm 9,3(154,0-187,0)$ \\
Tempo de Prática & $22,6 \pm 29,0(6,0-108,0){ }^{*}$ \\
(Meses) & $4,6 \pm 1,5(2,0-7,0)$ \\
Tempo Semana (Dias) & $82,9 \pm 32,7(60,0-150,0)$ * \\
Tempo por Dia & (Minutos) \\
\hline${ }^{*}$ p < 0,05 . Fonte: elaboração dos autores.
\end{tabular}

O ICP dos praticantes de kickboxing obtida neste estudo foi de 85,9 \pm $6,9 \%$ (Tabela 2), sendo que todos analisados apresentaram um ICP maior que $65 \%$, sendo assim, classificados como boa postura corporal ${ }^{8}$, resultados similares aos encontrados em outras práticas esportivas, como atletismo, hóquei sobre a grama, futebol, handebol e surf, que possui agilidade, força, equilíbrio, coordenação motora, capacidade cardiorrespiratória e velocidade de reação como valências físicas predominantes para o praticante ${ }^{10}$. Mesmo o grupo apresentando idade, tempo de prática em meses e dia por semana diferentes, não obteve diferença no ICP apresentado.

Detanico e colaboradores $^{12}$, também utilizando o PSU, verificaram a postura de 
praticantes de hóquei sobre a grama, obtendo resultados semelhantes ao do presente estudo, onde também tinha um grupo heterogêneo, com 31 atletas de ambos os sexos, com idade de 18 a 32 anos e variações no tempo de prática que chegou aos 16 anos; sendo que nenhum atleta esteve abaixo de $75 \%$, média que se encontra acima do índice recomendado pelo método para uma boa. Colaborando com os mesmos resultados, Santos e colaboradores $^{13}$, verificaram a postura de 40 atletas de atletismo, separando em dois grupos (adultos e adolescentes), os resultados mostraram ICP de 83,4\% $\pm 6,1$ \% para o grupo de atletas adultos e um ICP de $87,8 \pm 5,7 \%$ para o grupo de adolescentes, sendo que nenhum atleta apresentava ICP abaixo dos $75 \%$.

Tabela 2. Avaliação utilizando Portland State University (PSU)

\begin{tabular}{lc}
\hline Variáveis & $\begin{array}{c}\text { Média } \pm \text { Desvio Padrão } \\
\text { (Mínimo - Máximo) }\end{array}$ \\
\hline $\begin{array}{l}\text { Região cabeça e } \\
\text { pescoço - RCP (\%) }\end{array}$ & $76,9 \pm 9,3(60,0-92,0)$ \\
$\begin{array}{l}\text { Região coluna dorsal e } \\
\text { lombar - RCL (\%) }\end{array}$ & $86,2 \pm 9,5(73,0-100,0)$ \\
$\begin{array}{l}\text { Região abdominal e } \\
\text { quadril - RAQ (\%) }\end{array}$ & $91,9 \pm 10,8(73,0-100,0)$ \\
$\begin{array}{l}\text { Região membros } \\
\text { inferiores - RMI (\%) } \\
\text { Índice de correção } \\
\text { postural - ICP }(\%)\end{array}$ & $86,5 \pm 11,1(70,0-100,0)$ \\
\hline
\end{tabular}

Fonte: elaboração dos autores.

Com o objetivo de avaliar as alterações posturais de atletas de futebol de campo de uma equipe profissional, Santos e colaboradores ${ }^{14}$, também utilizaram o
PSU, com uma amostra maior, verificou, 116 atletas com idade entre 14 e 35 anos, e obteve resultados semelhantes ao do presente estudo. Corroborando com presente estudo, outros autores verificaram em atletas de hóquei $^{12}$ e atletismo ${ }^{13}$ que as variações de idade e tempo de prática não influenciam nos resultados do ICP detectadas em praticantes.

Verificando por regiões mensuradas, no presente estudo, as regiões da cabeça e pescoço (RCP; 76,9 \pm 9,3\%) apresentaram as menores médias percentuais entre as regiões. Resultados semelhantes foram encontrados no estudo de Peirão e colaboradores ${ }^{15}$, analisando surfistas amadores, onde o RCP também apresentou o menor percentual $(76,0 \pm 9,6$ \%) e o mesmo aconteceu em outro trabalho desenvolvido pelos mesmos autores ${ }^{16}$, com resultados parecidos encontrados em 17 surfistas profissionais, com a menor média sendo do RCP (83,5 $\pm \quad 8,5 \%)$. Corroborando, dados semelhantes também foram observados por Santos e colaboradores ${ }^{13}$, em uma análise feita com atletas de atletismo, com RCP de 85,5 \pm 8,3 $\%$.

No que diz respeito à classificação média dos segmentos corporais na vista dorsal e lateral, observamos que todos apresentavam alteração da altura dos ombros, $12(70,8 \%)$ alterações na coluna, 
$10 \quad(58,8 \%)$ na posição da cabeça e alterações no pé e $7(41,18 \%)$ alterações no joelho (Tabela 03).

Assim como neste estudo, Oliveira ${ }^{17}$ fez uma análise sobre alterações posturais em atletas de luta, em Muay Thai, onde selecionou 12 praticantes do sexo masculino, com idade média de 25 anos, encontrando resultados semelhantes ao do presente estudo, verificou que todos apresentaram algum tipo de alteração postural. O mesmo foi encontrando por Santos e colaboradores ${ }^{18}$, encontrando assimetrias corporais em 18 praticantes de Muay Thai, onde as mais acentuadas são nos ombros e coluna, resultados semelhantes ao presente estudo.

Tabela 3. Avaliação postural por segmento

\begin{tabular}{lc}
\hline Segmento & \multicolumn{1}{c}{$\mathbf{1 7 / n}(\%)$} \\
\hline Posição da Cabeça & $17 / 10(58,8 \%)$ \\
Altura do Ombro & $17 / 17(100,0 \%)$ \\
Alterações na Coluna & $17 / 12(70,8 \%)$ \\
Alterações no Joelho & $17 / 7(41,2 \%)$ \\
Alterações no Pé & $17 / 10(58,8 \%)$ \\
\hline
\end{tabular}

Fonte: elaboração dos autores.

Nas lutas, devido ao gesto técnico, essa postura alterada pode estar relacionada com a posição constante de rotação interna dos ombros e flexão dos membros superiores na posição de golpe e defesa, como descreve Santos e colaboradores ${ }^{18}$. Ademais, os movimentos das lutas promovem encurtamento do peitoral maior e um alongamento acentuado da região dorsal, podendo ocasionar uma má postura nesses atletas devido ao desequilíbrio muscular ${ }^{9}$.

Os autores $^{9,18}$ intensificam que as possíveis tendências posturais detectadas em praticantes de lutas podem contribuir para outros fatores, como uma maior ocorrência de lesões. Musculatura em padrões desejáveis pode evitar ou pelo menos minimizar lesões nas articulações e músculos nos seguimentos corporais, principalmente se essa preocupação com a postura iniciar nos atletas iniciantes para que futuramente não venham se prejudicar com a prática esportiva ${ }^{8,18}$.

Partindo desse pressuposto, a forma como se realiza o treinamento está diretamente associada ao surgimento dos desvios posturais, assim como a adaptação do corpo para cada modalidade praticada. Deste modo, faz-se necessário avaliar além da intensidade e volume do treinamento, as correções relacionadas ao gesto técnico decorrente da prática esportiva, objetivando melhorar o rendimento do lutador e prevenir lesões que possam ocorrer relacionadas às características posturais apresentadas nas práticas esportivas ${ }^{19}$.

Destaca-se o fato do estudo abrir discussões acerca de aspectos posturais em praticantes de Kickboxing, possibilitando melhorias no processo de treinamento da modalidade. Entretanto, algumas limitações 
devem ser citadas, como por exemplo, o bi dimensionamento oriundo das imagens analisadas no plano 2D. Assim como, o registro manual das imagens, em oposição ao registro automático.

\section{CONCLUSÃO}

Embora a análise postural utilizando o método PSU não tenha identificado desvios posturais acentuados, os resultados de alinhamento corporal mostram desvio principalmente na altura dos ombros, Desta forma, observou-se que o padrão exigido pela prática do kickboxing pode levar a uma postura alterada e, segundo a literatura $^{8,9,18}$, aumentar o risco de lesões e reduzir o desempenho. Com isso, verificamos a importância com o cuidado da postura dos praticantes de kickboxing, na perspectiva de melhorar o desempenho e reduzir o risco de lesões.

\section{REFERÊNCIAS}

1. ZORELLO, P. Qualidade Total Kick Boxing Brasil, introdução. São Paulo Confederação Brasileira de KickBoxing, 2007. (Apostila)

2. LIMA, F. R.; PINTO, A. L. S.; PEREIRA, R. M. E.; CAPARBO, V. F.; SOUZA, M.; GUALANO, B. Incidência e fatores de risco de lesões osteomioarticulares em corredores: um estudo de coorte prospectivo. Rev. Bras. Educ. Fís. Esporte, 2010; 24:453-462.

3. MAGEE D. J. Avaliação Postural In: Disfunção Musculoesquelética. 3. ed., São Paulo: Manole, 2002; 105-157.

4. PALMER, L. M.; APLER, M. E. Postura. In: Palmer, L. M; Apler, M.E.
Fundamentos das Técnicas de Avaliação Musculoesquelética. 2 edição, São Paulo: Guanabara Koogan. 2000; 195-212.

5. AMTMANN, J. A. Self-reported training methods of mixed martial artists at a regional reality fighting event. J Strength Cond Res. 2004;18:194-196.

6. TAMBORDEGUERI, A. C.; TIRLONI, A. S.; DOS REIS, D. C. Incidências de lesões e desvios pulmonares em atletas de taekwondo Rev Bras. Ciênc. Esporte, Florianópolis, v 33, n 4, 2011; 975 - 990.

7. FLECK, S. J.; KRAEMER, W. J. Fundamentos do Treinamento de Força Muscular, ArtMed, 3 edição. 2007; 50-62.

8. SANTOS, J. B.; MORO, A. R. P.; CEZAR, M. R.; REIS, P. F.; LUZ, J. D.; REIS, D. C. Descrição do Método de avaliação postural de Portland State University. Rev Fisioter Bras. 2005; 6 (5) atualizações.

9. KENDALL, F. P.; MCCREARY, E. $\mathrm{K}$.; PROVANCE, P. G. Postura: alinhamento e equilíbrio muscular. In: KENDALL，F. P.; MCCREARY， E. K; PROVANCE, P. G. Músculos Provas e Funções. $4^{\mathrm{a}}$ edição, São Paulo: Manole, 2007; 69-118

10. GOMES, M. S. P. O ensino do saber lutar na universidade: estudo da didática clínica nas Lutas e Esportes de Combate. 204f. Tese (doutorado em Educação Física) Faculdade de Educação Física. Universidade Estadual de Campinas, Campinas, 2014.

11. GOMES, F. P. Curso de estatística experimental. Piracicaba: Nobel, 1990.

12. DETANICO, D.; REIS, D. C.; CHAGAS, L.; SANTOS, S. G. Alterações posturais, desconforto corporal (dor) e lesões em Atletas das seleções brasileiras de hóquei sobre a grama. R. da Educação Física/UEM. Maringá, v. 19, n. 3, p. 423430, 3. trim. 2008.

13. SANTOS, J. B.; DUBARD, M.; RODRIGUES, S. S.; DUBARD, M. A. Alterações posturais de atletas de atletismo de alto rendimento. Revista Brasileira de 
Fisiologia do Exercício - Volume 12 Número 4 - julho/agosto 2013.

14. SANTOS, J. B.; TOLEDO, E.; REIS, P. F.; MORO, R. P.; GOMES, A. C. Alterações posturais em atletas de futebol de uma equipe profissional na faixa etária entre 14 a 35 anos de idade. Revista Brasileira de Prescrição e Fisiologia do Exercício, São Paulo. v.9. n.50. p.772-781. Nov./Dez. 2014. ISSN 1981-9900.

15. PEIRÃO, R.; TIRLONI, A. S.; REIS, D. C.; COELHO, P. B.; GUGLIELMO, L. G. A. Incidência de Desvios posturais em surfistas amadores. Em: Congresso de ciências do desporto e educação física dos países de língua portuguesa, XII, 2008, Porto Alegre. Anais do XII Congresso de ciências do desporto e educação física dos países de língua portuguesa. Porto Alegre: UFRGS; 2008.

16. PEIRÃO, R.; TIRLONI, A. S.; REIS, D. C. Avaliação postural de surfistas profissionais utilizando o método Portland State University (PSU). Fit Perf J. 2008, nov-dez;7 (6):370-4

17. OLIVEIRA, W. S.; SOBRINHO, J. Q. Avaliação postural em praticantes de muaythai no município de horizonte/ce.. In: Anais da VII Mostra de Pesquisa em Ciência e Tecnologia DeVry Brasil. Anais... DEVRY BRASIL, 2016. Disponível em: <https//www.even3.com.br/anais/viimostrad evry/28957-AVALIACAO-POSTURALEM-PRATICANTES-DE-MUAYTHAINO-MUNICIPIO-DE-HORIZONTECE $>$. Acesso em: 02 de Out. 2017 22:51

18. SANTOS, R. V.; DA VEIGA. R. A. D. R. Avaliação postural de praticantes da Arte marcial muaythai no município de Erechim/RS. PERSPECTIVA, Erechim. v.36, n.133, p.163-178, março/2012.

19. SOUSA, B. R. G.; TEIXEIRA, D. O. T; SABINO, G. S. Aplicação da avaliação funcional de Movimento (FMS) em praticantes de Muay thai de BELO HORIZONTE/MG. Revista Interdisciplinar Ciências Médicas - MG 2017, 1(1): 51-61.
20. DEZAN, V. H.; SARRAF, T. A.; RODACKI, A. L. F. Alterações posturais, desequilíbrios musculares e lombalgias em atletas de luta olímpica. R. Bras. Ci e Mov. Brasília v. 12 n.1, 2004;35-38.

21. LEROUX, J. L.; CODINE, P.; THOMAS, E.; POCHOLLE, M.; MAILHE, D.; BLOTMAN, F. Isokinetic evaluation of rotational strength in normal shoulders and shoulders with impingement syndrome. Clin Orthop Relat Res. 1994;304:108-115.

22. ZORELLO, P. Historia do Kick Boxing. Rio de Janeiro: APKB, 1992. Disponível em: <http://www.cbkb.com.br/site/cbkb/historia kickboxing>. Acesso em: 01 out. 2017.

Informações do artigo / Information of this article:

Recebido: 12/09/2018

Aprovado: 15/01/2019

Publicado: 07/05/2019

Received: 12/09/2018

Approved: 15/01/2019

Published: 07/05/2019

Wlaldemir Roberto dos Santos

ORCID: $\quad$ https://orcid.org/(0000-0001-5706$\underline{2243}$.

Contribuição dos autores

Todos os autores participaram do desenvolvimento e execução do estudo, assim como das análises e redação do manuscrito.

Conflito de interesses

Os autores declaram não haver conflito de interesses.

Como citar este artigo:

Dos Santos, A. J.; dos Santos, W. R.; Paes, P. P.; dos Santos, W. R. Análise Postural dos Praticantes de Kickboxing. Arq. Bras. Ed. Fís., Tocantinópolis, v. 2, n. 1, Jan./Jul., p. 21 - 29, 2019. 